\title{
THE RELATIONSHIP OF SODIUM CHLORIDE TO HYPERTENSION ${ }^{1}$
}

\author{
By GEORGE A. PERERA ANd DAVID W. BLOOD \\ (From the Department of Medicine, Columbia University College of Physicians and Surgeons, \\ the Presbyterian Hospital, and the Research Service, First [Columbia] Division, \\ Goldwater Memorial Hospital, Department of Hospitals, New York City)
}

(Received for publication May 15, 1947)

Restriction of the sodium chloride intake as a therapeutic measure in hypertensive vascular disease was first advocated many years ago $(1,2$, 3). Although subsequent investigators (4, 5, 6, 7) claimed that the addition or removal of salt from the diet failed to influence the blood pressure level significantly, interest in the subject has recently been renewed.

Selye and his coworkers (8) noted a striking hypertensive effect when sodium chloride was administered to experimental animals receiving injections of desoxycorticosterone acetate. Grollman and his associates (9) observed that drastic reduction in sodium intake resulted in a decline in blood pressure in some hypertensive patients. They believed it probable that the beneficial effects of the diet proposed by Kempner (10) might be due to the restriction of salt. Recently evidence has been presented (11) that sodium chloride potentiates the pressor activity of desoxycorticosterone acetate when injected into rats rendered nephritic with nephrotoxic serum.

The present study was undertaken to reexamine the relationship of sodium chloride to hypertensive vascular disease in man.

\section{METHODS}

Men and women with uncomplicated hypertensive vascular disease were studied on the wards of the Presbyterian Hospital and the Research Service, First Division, of the Goldwater Memorial Hospital. Subjects were included only if the antecedent blood pressure consistently exceeded 140/90 mm. of mercury and in the absence of cardiac pain or insufficiency, renal or cerebral complications or fever. All patients were free of albuminuria, showed normal phenolsulfonephthalein excretion and

1 This study was made possible through the generosity of the Albert and Mary Lasker Foundation. We are indebted to Miss Ann D. Barrows, dietitian, Miss Margaret G. Hawthorne, head nurse, Miss Katherine Vislocky, technician, John T. Sharp and Dominic G. Iezzoni for their invaluable assistance. urine concentration tests, and in all instances the venous pressure was within normal limits.

Blood pressures were measured each morning in the same arm by the same observer, with the subject quiet and relaxed in bed, the head of which was raised to a $30^{\circ}$ angle. At least 5 and usually 7 or 8 readings were taken at half-minute intervals and the lowest systolic and diastolic value recorded. This value was designated the "resting" blood pressure as compared to "casual" readings taken under various conditions of activity at other times during the day. In order to secure an adequate baseline, it was found obligatory to carry out preliminary observations for at least 3 weeks.

All subjects were kept in bed until after blood studies and blood pressure measurements were made, and ambulatory activity was standardized at a constant level the rest of each day. The patients were weighed daily before breakfast on the same scales. The daily fluid intake and urine output were recorded, distilled water being supplied for drinking purposes.

Throughout the study the patients were given a constant diet and fluid intake. Identical salt-poor daily menus were prepared. Repeated direct analyses of aliquots taken from an entire day's cooked diet gave values of between 0.25 to 0.35 grams of sodium or considerably less than 1 gram of sodium chloride per day. These salt-poor diets yielded 1,700 to 2,200 calories and 70 to 80 grams of protein, and did not include special ingredients such as dialyzed milk. Sodium chloride was administered by mouth using weighed salt shakers, additional supplements being given in some instances in the form of enteric-coated tablets. When indicated, desoxycorticosterone acetate 2 (DCA) was injected subcutaneously in doses of $5 \mathrm{mgm}$. twice daily.

The urine chloride was determined daily from the 24hour collection, daily aliquots being measured at approximately weekly intervals for their sodium content. Fasting blood samples were obtained at intervals for $\mathrm{CO}_{2}$ content, chloride, sodium, potassium, protein and urea nitrogen determinations. The serum volume was measured with the blue dye T. 1824. Weekly electrocardiograms were taken and in no instance showed evidence of significant myocardial damage or serial changes.

A sample chart (Figure 1) illustrates the general form

2 Desoxycorticosterone acetate (Doca) was supplied through the courtesy of Dr. L. A. Pirk of Roche-Organon, Inc., Nutley, New Jersey. 


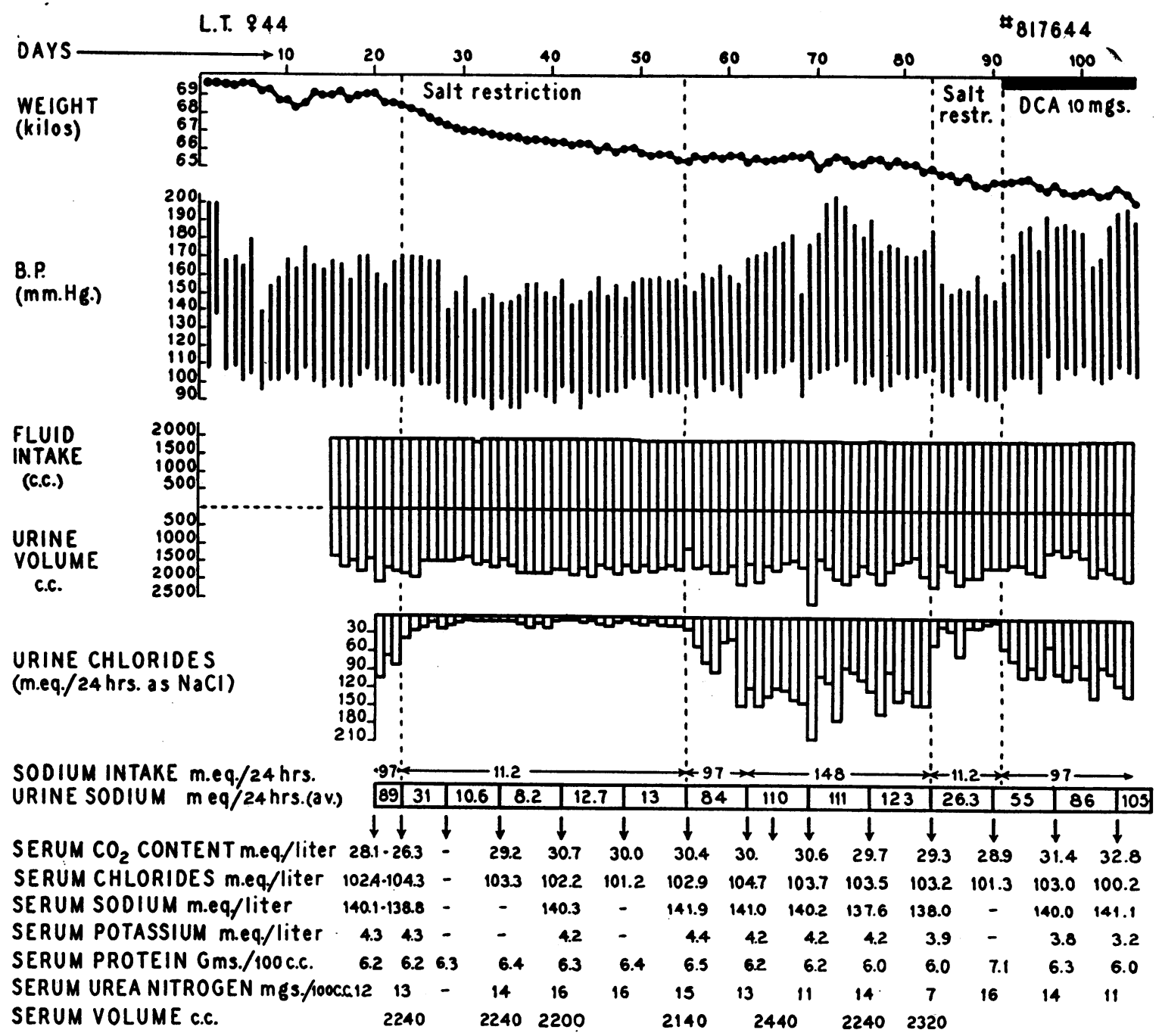

Fig. 1. Sample Chart Illustrating General Form of Investigations on Each Hypertensive Subject

in which subsequent investigations were conducted and recorded on each patient.

\section{RESULTS}

I. Effect of rigid sodium chloride restriction on blood pressure. Six hypertensive patients were studied following a preliminary baseline period and constant regimen which included 4 grams of sodium chloride added to the salt-poor diet. A decrease in "resting" blood pressure was observed in all patients in from 3 to 5 days after the withdrawal of the sodium chloride from the diet, with a return to previous levels only after sodium chloride administration was resumed (Fig- ure 2, I). The majority of blood pressure readings, during the period of observation, remained above 140/90 mm. of mercury. "Casual" readings were apparently uninfluenced by sodium chloride restriction, and each patient exhibited the same variation, the same response to such stimuli as activity, apprehension or the cold pressor test as was demonstrated during the foreperiod.

The withdrawal of sodium chloride from the diet for a period of 2 weeks was tolerated in these subjects without discomfort. Slight weight loss and small increases in urine output were generally observed. The decreased intake was sharply 

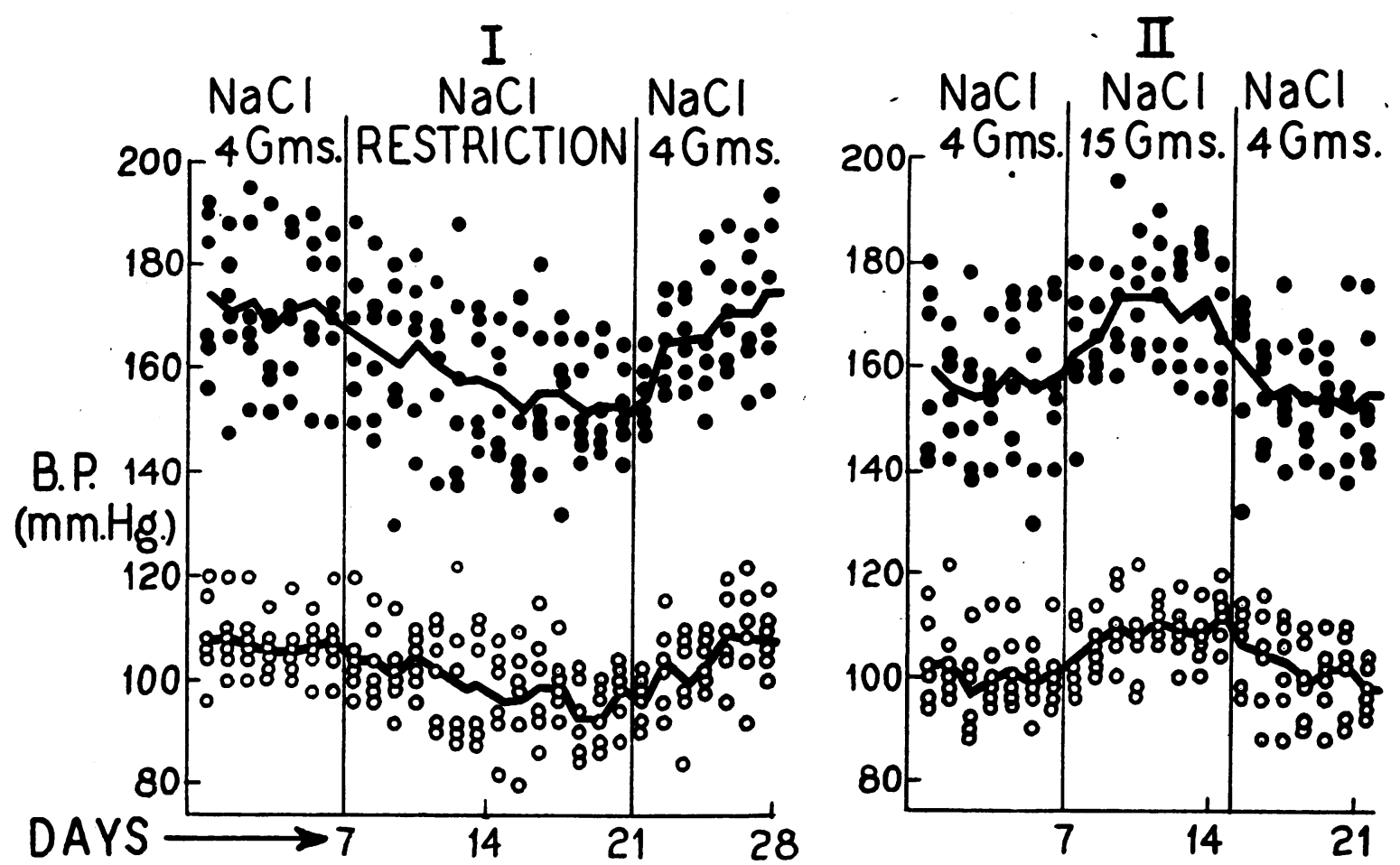

Fig. 2. Effect of Rigid Sodium Chloride Restriction and of Increased Sodium Chloride Intake on 6 Patients with Hypertension

reflected in urine sodium and chloride levels, but serum $\mathrm{CO}_{2}$, chloride, sodium, potassium, protein, urea nitrogen and volume measurements were not materially influenced (Table I). Ballistocardiographic tracings were unchanged on repeated records taken before and after sodium chloride restriction in 2 of these patients, both of whom exhibited a significant drop in blood pressure on salt restriction.

II. Effect of increased sodium chloride intake on blood pressure. Six hypertensive patients, 4 of whom had been included in the previous study, were investigated following a preliminary baseline period and constant regimen which included 4 grams of sodium chloride added to the salt-poor diet. The intake of sodium chloride was then increased to 15 grams daily for an 8-day period. These subjects were able to tolerate this dosage without gastric intolerance or diarrhea.

A rise in "resting" blood pressure was observed in 5 patients within 1 to 4 days after the addition of sodium chloride to the diet, with a return to previous levels after the initial dosage was resumed (Figure 2, II). (In 2 patients a week's trial with placebo pills was without effect on the blood pressure.) Again "casual" readings were uninfluenced and each patient exhibited the same variation, the same response to such stimuli as activity, apprehension or the cold pressor test as was demonstrated before the increased sodium chloride intake.

The further addition of sodium chloride for a period of 8 days was followed by an increase in urine sodium and chloride excretion, and slight weight gain and decreases in urine output were generally observed. No significant change was recorded in serum $\mathrm{CO}_{2}$, chlorides, sodium, potassium, protein, urea nitrogen, or volume measurements (Table II).

III. Effect of rigid sodium chloride restriction 
TABLE I

Clinical and laboratory data of 6 hypertensive patients before and after rigid sodium chloride restriction

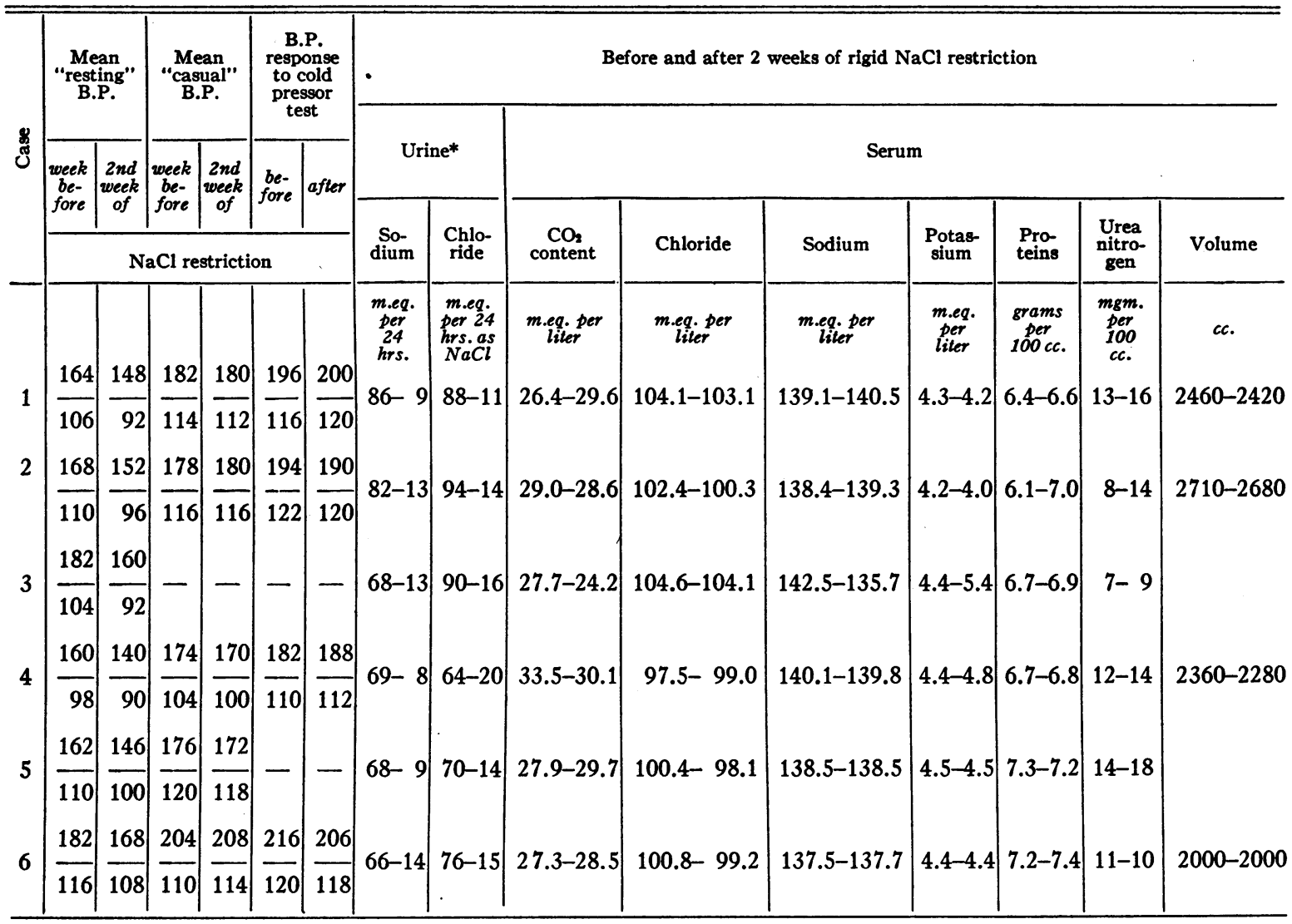

* Mean values for each period.

on pressor action of $D C A$ (12). Five hypertensive patients, 3 of whom had been investigated in the previous studies, were studied following a preliminary baseline period and constant regimen which included 4 grams of sodium chloride added to the salt-poor diet. DCA was then injected subcutaneously in doses of $5 \mathrm{mgm}$. twice daily, with added dietary sodium chloride increased in 3 subjects but maintained at 4 grams in 2 . After 10 days, all added sodium chloride was withdrawn from the diet without other change in regimen and while the subjects continued to receive DCA.

In all instances an increase in "resting" blood pressure followed the injection of DCA, this rise not being maintained when sodium chloride was withdrawn (Figure 3 ). During the period of sodium chloride and DCA administration, the expected slight increases in weight, hemodilution and serum volume were generally noted, together with slight reduction in urine volume $(12,13)$. Significant increases in serum chloride and sodium were observed in 2 patients, while reductions in serum potassium of from 0.4 to $1.5 \mathrm{~m}$.eq. took place in all subjects. When DCA injection was continued.but with rigid sodium chloride restriction, slight reductions in weight and serum volume were seen, together with hemoconcentration, slight increases in urine volume, and a sharp decrease in urine sodium and chloride values. The serum chloride and sodium were significantly. reduced by sodium chloride restriction in 2 instances, while the serum potassium levels showed 
TABLE II

Clinical and laboratory data of 6 hypertensive patients before and after increased sodium chloride intake

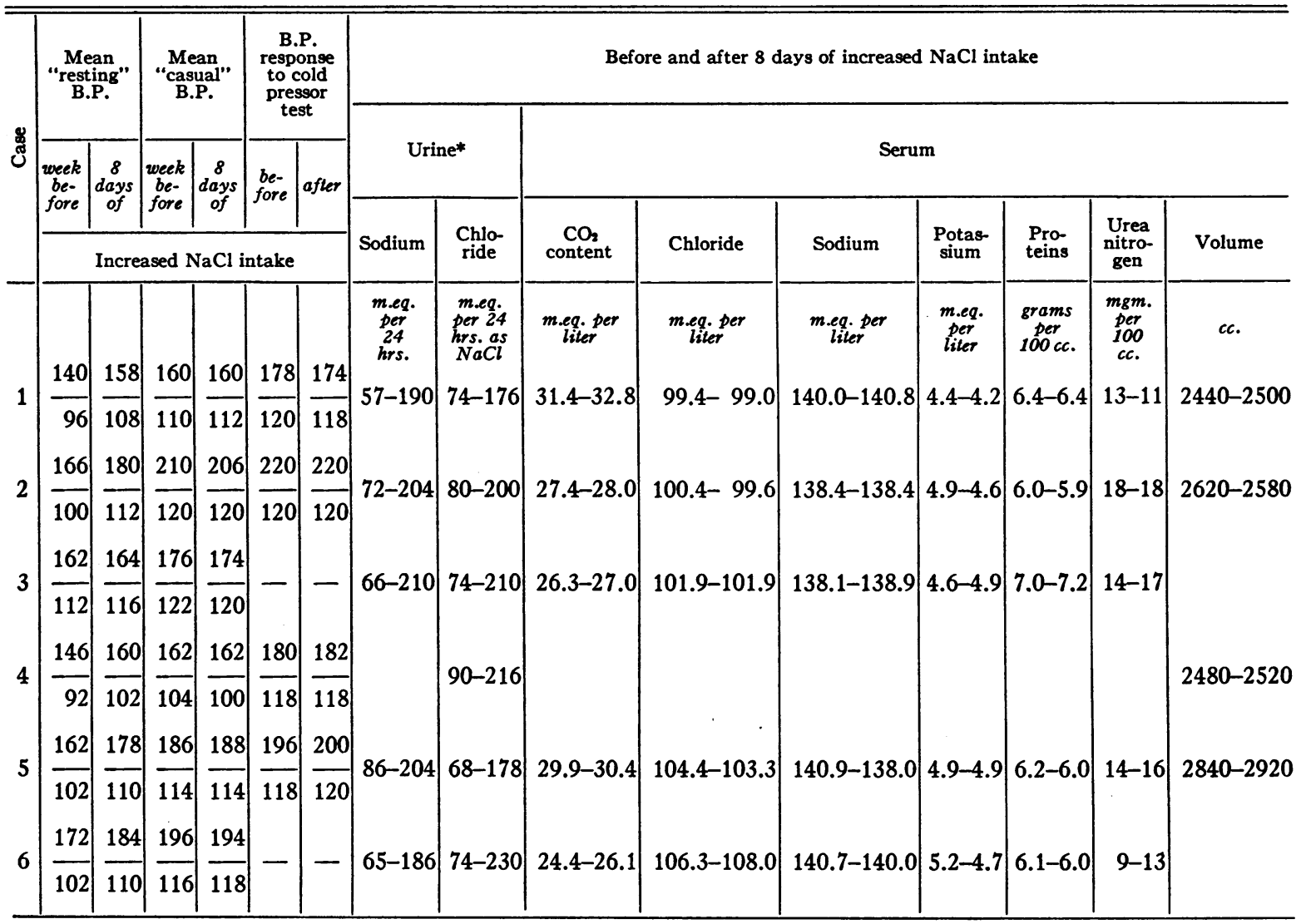

* Mean values for each period.

no further consistent change (Figure 4, Table III).

As the pressor action of prolonged DCA injection was on occasion found to be transitory, the order was reversed in 2 additional patients, i.e., following the usual baseline observations, rigid sodium chloride restriction was instituted simultaneously with the start of DCA administration. These were patients who had not been subjected to previous studies. No rise in "resting" blood pressure ensued until the addition of sodium chloride (Figure 5).

\section{COMMENT}

The present study, utilizing measurements of the "resting" blood pressure, confirms the reports of some previous observers that the rigid restriction of sodium chloride in the diet may lower the blood pressure of patients with hypertensive vascular disease. Conversely, it has been shown under similar conditions that large amounts of dietary sodium chloride may exert a pressor effect in hypertensive subjects. Lastly, the observations of Knowlton and her associates (11), that sodium chloride participates in the potentiation of the pressor activity of DCA, have been supported by studies in patients with an elevated blood pressure. It has been previously shown that this pressor response to DCA is not due to salt and water retention alone (12).

Because of the great variability of blood pressure readings in association with any of a large 
number of environmental changes, the need has been stressed for an adequate baseline and constant regimen as well as for the differentiation between "resting" and "casual" values. It must be em-

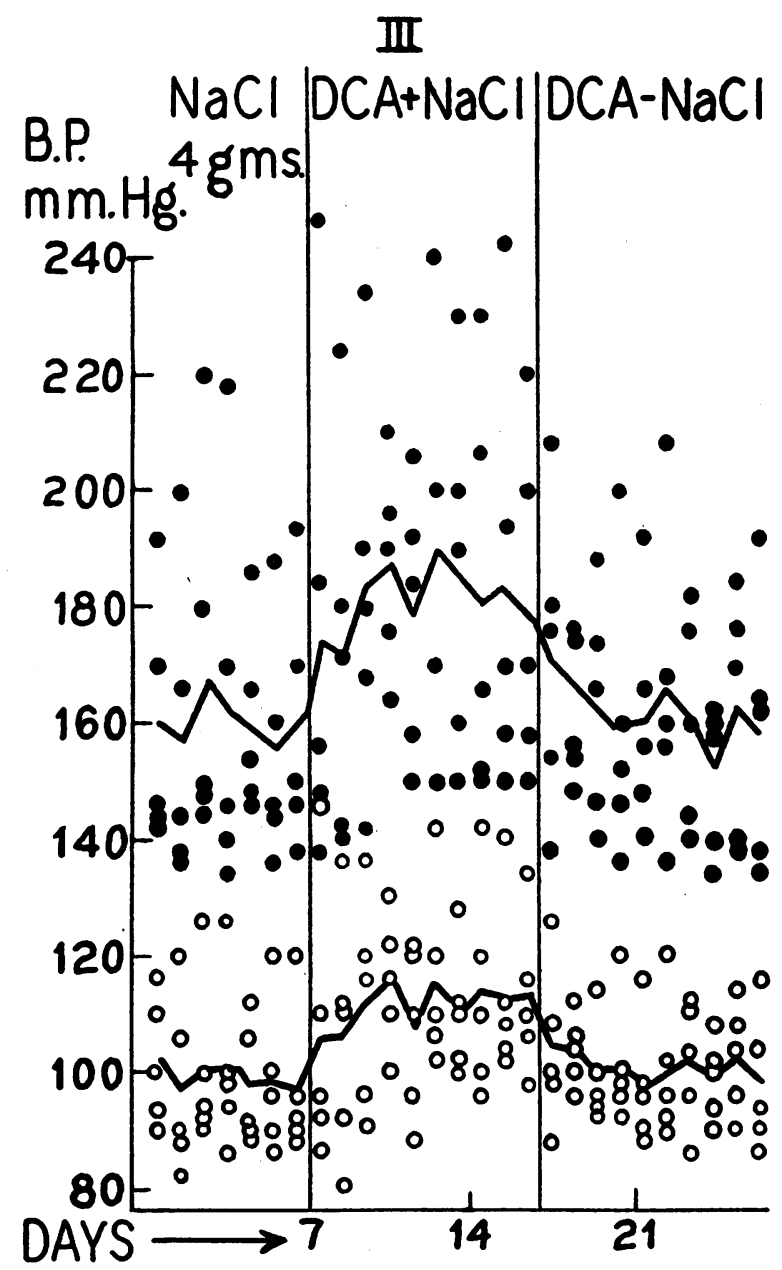

Fig. 3. Effect of Rigid Sodium Chloride Restriction on Pressor action of DCA in 5 Hypertensive SubJects

phasized that the magnitude of blood pressure fluctuation observed in this study was often small, although consistent, and that standard conditions were required.

The effect of rigid sodium chloride restriction is apparently unrelated to changes in circulatory volume or cardiac output, hence must be concerned with alterations in peripheral resistance. It must be noted that a normal blood pressure is not routinely achieved by salt restriction and that the patient's ability to respond to autonomic or neuro- genic stimuli is unaffected. The demonstration in this study that there is an absence of correlation between "resting" and "casual" blood pressure readings, suggests that the factors (at least in part neurogenic) influencing the "casual" readings may be independent of those influenced by sodium chloride or DCA. This may explain the results of sympathicolytic drugs and of surgical procedures which modify vasoconstriction of sympathetic origin yet do not invariably relieve the hypertensive state. Previous uncertainties concerning the effect of many therapeutic agents may have been due to the failure to dissociate between extrinsic (neurogenic) and direct actions on peripheral resistance, both of which seem to be involved-in variable proportions-in the clinical picture of hypertension.

There is nothing to suggest that the small changes in "resting" blood pressure associated with rigid sodium chloride withdrawal are of therapeutic significance or that prolonged restriction will exert any influence on the natural history of this disorder.

The modification of "resting" blood pressure levels by sodium chloride and the relationship of sodium chloride to the pressor action of DCA are consistent with the view that some product of the adrenal cortex may play a part in the mechanism of hypertensive vascular disease $(12,14,15)$.

\section{CONCLUSIONS}

1. Observations have been made on patients with uncomplicated hypertensive vascular disease under controlled conditions of diet and activity.

2. Despite an otherwise constant regimen, rigid sodium chloride restriction resulted in a slight decrease in "resting" blood pressure in 6 subjects, whereas large amounts of dietary sodium chloride produced a slight rise in "resting" blood pressure in 5 of 6 subjects.

3. The pressor action of desoxycorticosterone acetate was observed in 7 subjects, but only when sodium chloride was included in the diet; the rigid restriction of sodium chloride obliterated this pressor response.

4. It is suggested that vasoconstrictive factors (at least in part neurogenic) in hypertensive patients are independent of those alterations in peripheral resistance influenced by sodium chloride or desoxycorticosterone acetate. 
M.B. $₹ 37$

\#842993

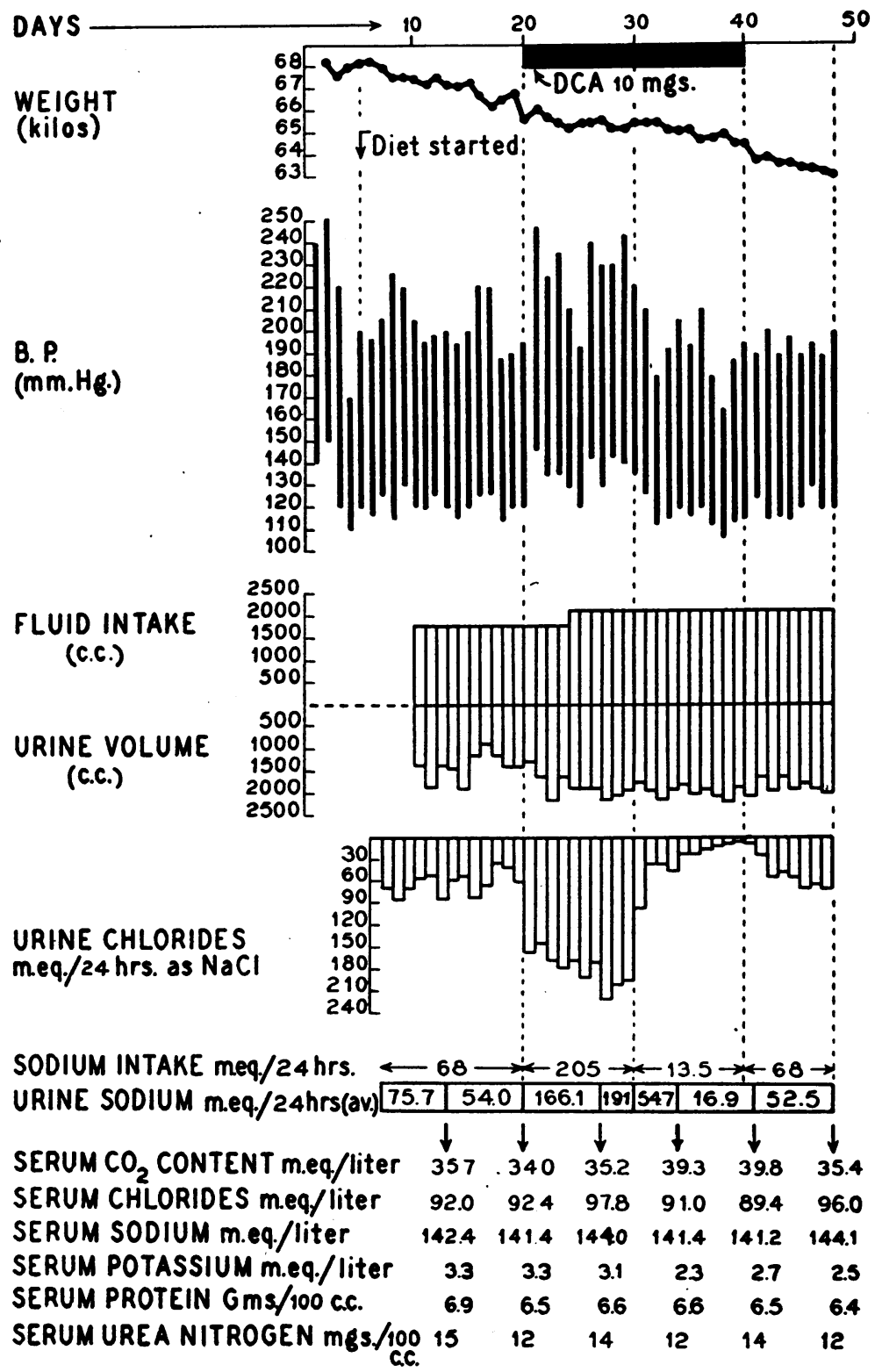

Fig. 4. Clinical and Laboratory Data Illustrating Effect of Rigid Sodium Chloride Restriction on Pressor Action of DCA 


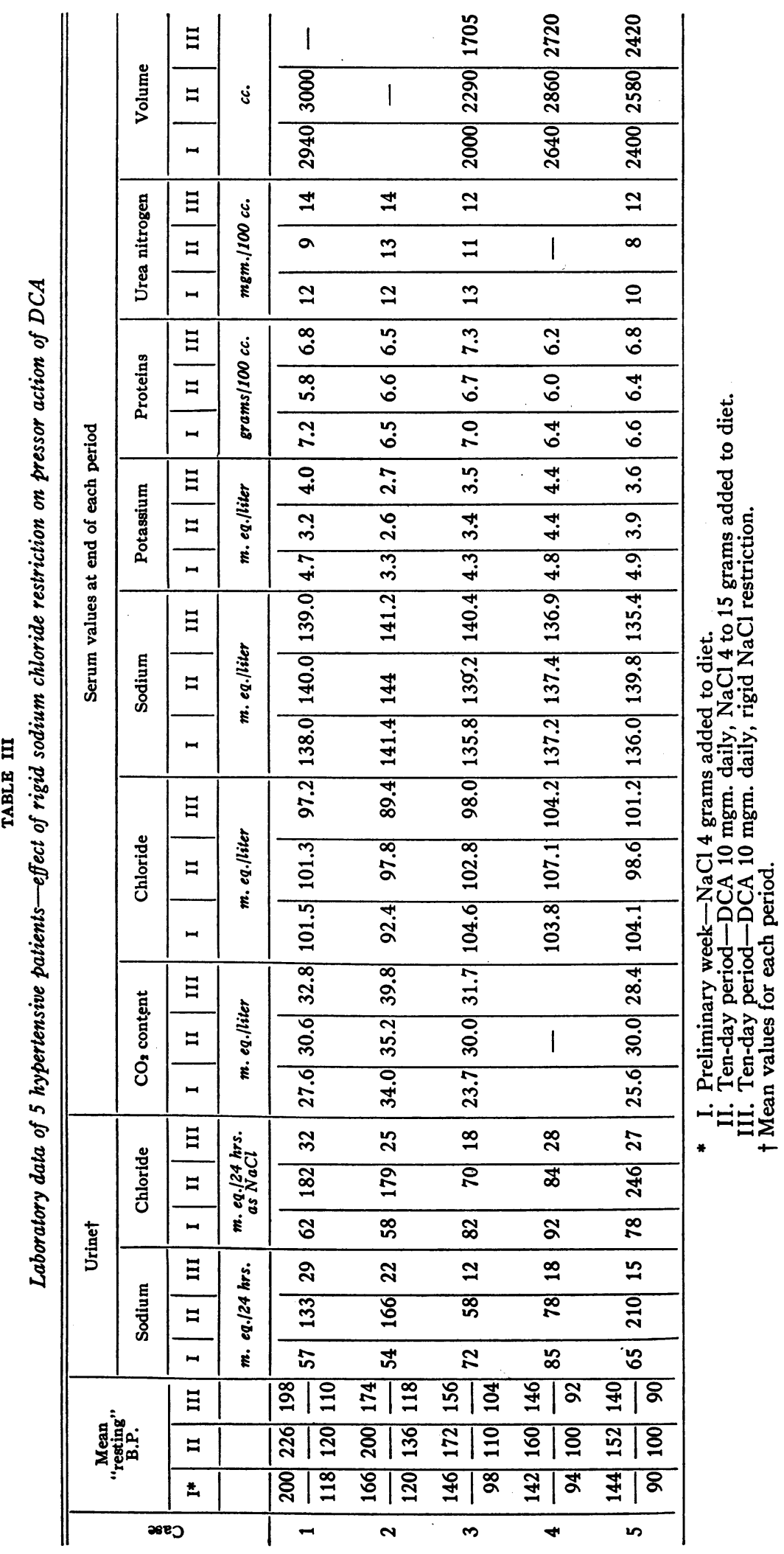




\section{$R C \& 44$}

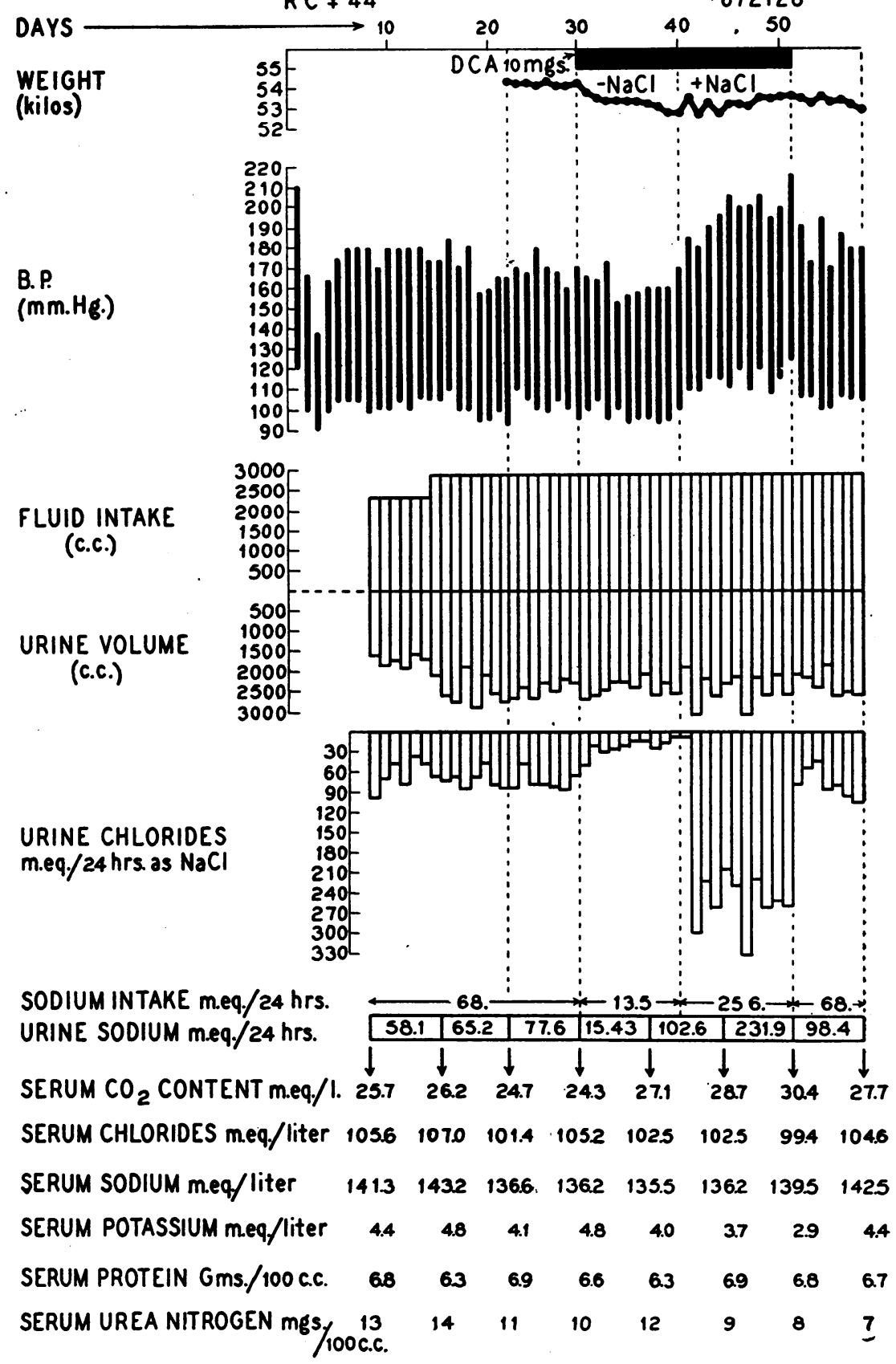

Fig. 5. Clinical and Laboratory Data Illustrating Effect of Rigid Sodium Chloride Restriction on Pressor Action of DCA 
5. The relationship of sodium chloride and desoxycorticosterone acetate to hypertensive vascular disease is consistent with the view that some product of the adrenal cortex may play a part in the mechanism of this disorder.

\section{BIBLIOGRAPHY}

1. Volhard, F., Die Behandlung der Sklerosen, in von Bergmann, G., and Staehelin, R., Handbuch der inneren Medizin, Berlin, Julius Springer, Vol. 6, Pt. 2, p. 1753, 1931, Ed. 2.

2. Ambard L., and Beaujard, E., La rétention chloruée sèche. Semaine méd., 1905, 25, 133.

3. Allen, F. M., Arterial hypertension. J. A. M. A., $1920,74,652$.

4. Mosenthal, H. O., The treatment of high blood pressure. M. Clinics N. America, 1922, 5, 1139.

5. McLester, J. S., The influence of rigid salt restriction in the diet of chronic nephritis. Am. J. M. Sc., 1922, 163, 794.

6. O'Hare, J. P., and Walker, W. G., Salt in vascular hypertension. Arch. Int. Med., 1923, 32, 283.

7. Berger, S. S., and Fineberg, M. H., The effect of sodium chloride on hypertension. Arch. Int. Med., 1929, 44, 531.

8. Selye, H., Hall, C. E., and Rowley, E. M., Malignant hypertension produced by treatment with desoxy- corticosterone acetate and sodium chloride. Canad. M. A. J., 1943, 49, 88.

9. Grollman, A., Harrison, T. R., Mason, M. F., Baxter, J., Crampton, J., and Reichsman, F., Sodium restriction in the diet for hypertension. J. A. M. A., 1945, 129, 533.

10. Kempner, W., Treatment of kidney disease and hypertensive vascular disease with rice diet. North Carolina M. J., 1944, 5, 125.

11. Knowlton, A. I., Loeb, E. N., Stoerck, H., and Seegal B. C., Desoxycorticosterone acetate: the potentiation of its activity by sodium chloride. J. Exp. Med., 1947, 85, 187.

12. Perera, G. A., and Blood, D. W., The pressor activity of desoxycorticosterone acetate in normotensive and hypertensive subjects. Ann. Int. Med., 1947, $27,401$.

13. Clinton, M., Jr., and Thorn, G. W., Effect of desoxycorticosterone acetate administration on plasma volume and electrolyte balance of normal human subjects. Bull. Johns Hopkins Hosp., 1943, 72, 255.

14. Perera, G. A., The relationship of the adrenal cortex to hypertension: observations on the effect of hypoadrenalism on a patient with hypertensive vascular disease. J. A. M. A., 1945, 129, 537.

15. Perera, G. A., and Blood, D. W., Disturbance in salt and water metabolism in hypertension. Am. J. Med., 1946, 1, 602. 